\title{
Structural features of complex hydrochemical systems
}

\author{
Olga Shevtsova ${ }^{1, \star}$ \\ ${ }^{1}$ V.I. Il'ichev Pacific Oceanological Institute FEB RAS, Vladivostok, Russia
}

\begin{abstract}
The set of non-conservative hydrochemical parameters is considered as a complex system, which displays collective behavior. It is found that the collective behavior is described by the power relation between the time variability (the standard deviations) and the average concentrations of different hydrochemical parameters in the scale range $100-0.0001 \mathrm{mg} / \mathrm{kg}$. The exponent can be $0.7-0.9$. Power law scaling is the mathematical expression of self similarity and fractality. The complex systems of nonconservative chemical parameters have a structure that can be characterized by exponent, normalization coefficient, standard error, correlation coefficient, and by sharp deviations of the individual parameters from the regression line and from the most probable average and standard deviation values, if any. It is shown with specific examples that changes in the hydrochemical systems structure are the result of the manifestation of biogeochemical processes and the dynamics of water. Regression analysis of collective behavior of complex hydrochemical systems is one of the examples of the use of modern information technologies based on the methods of system analysis.
\end{abstract}

\section{Introduction}

The set of various non-conservative hydrochemical parameters can be considered as a complex system $[1,2]$.

A complex system is a system composed of many interacting parts, which displays collective behavior that does not follow trivially from the behaviors of the individual parts. Examples of complex systems can be found in various areas of natural sciences (seismology, astrophysics, turbulence theory, allometry, ecology, etc.), and they are still poorly understood [3]. The collective behavior in a number of complex systems is described by the power law. The observation and origin of power laws and scaling in complex systems has been a subject of discussion and research for many decades. Power law scaling is the mathematical expression of self similarity and fractality [1-6].

The structure of complex systems whose collective behaviors obeys a power law can be described by the regression characteristics: its parameters, standard error, correlation coefficient, and sharp deviations of the individual system parts from the regression line and from the most probable values of the parts characteristics, if any. Comparison of the regression characteristics for the similar complex systems under different observation conditions can serve as the basis for the studying of its characteristic features and dynamics. It is of interest to consider on specific examples how observations conditions can affect structure of complex systems.

\footnotetext{
${ }^{\star}$ Corresponding author: OV_shevtsova@mail.ru
} 
It has already been shown earlier on the example of the Amur Bay (Sea of Japan) that the collective behavior of the hydrochemical system manifests itself as the power dependence between the time average concentrations $\langle P\rangle$ and the time variability $s$ (standard deviations) of various non-conservative hydrochemical parameters:

$$
\log s=\gamma \log \langle P\rangle+\log \xi
$$

where $\gamma$ is the exponent, $\xi$ is the normalization coefficient. This fact was registered on the basis of the time series data of 9 hydrochemical parameters in the scale range $0.0001-100 \mathrm{mg} / \mathrm{kg}$ obtained using standard methods at 13 points of four daily stations in various parts of Amur Bay. Estimates of the regression characteristics and the sample standard deviation of the parameter estimates $\pm \mathrm{SD}$ were as follow: $\gamma=0.68 \pm 0.02 ; \xi=0.111 \pm 0.007$; correlation coefficient $r=0.97$; standard error $s_{y x}=0.28$ (number of data points $n=102$ ) [1,2,4]. The slope $\gamma$ characterizes the system behavior on the whole. Coefficient $\xi$ is the ratio of $s$ to $\langle P\rangle^{\gamma}$ for all chemical parameters of system. It results from (1) that $\xi=s /\langle P\rangle^{\gamma}$.

\section{Hydrochemical system in the Ezcurra Inlet}

The Ezcurra Inlet constitutes part of Admiralty Bay, situated in the southern region of King George Island (South Shetland Islands). The power dependence (1) obtained this paper after a preliminary analysis of time-series nitrites, phosphates, nitrates, silicate, and oxygen data is represented in Fig. 1. The observations were made on board the anchored ship during the Polish Academy of Sciences Second Antarctic Expedition during part of the Antarctic summer, when the fluctuations of salinity amounted to $\pm 0.2^{0} / 00$ with respect to the weighted average of $33.9^{0} \%$ and the temperature varied by $\pm 1^{0} \mathrm{C}$ with respect to the mean value of $+0.2^{0} \mathrm{C}[7-8]$.

We have analyzed observations throughout January 1 - March 10, 1978. The numbers of measurements used to estimate $s$ and $\langle P\rangle$ were 28-49 at 1, 5, 10, 50, $60 \mathrm{~m}$ and 9-21 at 20,30, 40, 70 $\mathrm{m}$. Only chemical parameters with time variations significant at the $95 \%$ level were used. The final equation of a regression $s$ against $\langle P\rangle$ and $\pm \mathrm{SD}$ of the parameter estimates are:

$$
\log s=(0.760 \pm 0.015) \log \langle P\rangle+\log (0.037 \pm 0.001), \quad n=24, \quad r=0.996 .
$$

In the Ezcurra Inlet, structural features of complex hydrochemical system were as follows (Fig. 1):

1. The parameter estimate $\gamma=0.76<1$;

2. Strong correlation coefficient $r$;

3. Unusually small scattering of points around the final regression line, $s_{y x}=0.060 \mathrm{mg} / \mathrm{kg}$;

4. Very small normalization coefficient $\xi=0.037$;

5. The sharp deviation of dissolved oxygen dots in bottom water from the regression line: 103, 127 , and $167 \%$ at 50, 60, and $70 \mathrm{~m}$ corresponding;

6. The time variations of nitrites at all depths were statistically significant at no more than $50 \%$ level. They were almost completely determined by the observation accuracy $14 \%$ [8].

The structural features of the hydrochemical system in the Ezcurra Inlet are a consequence of mainly the hydrological regime. Ideal conditions for the vertical mixing of water masses exist here. The fiord is a narrow and shallow reservoir. In the measuring area, it is about $2.1 \mathrm{~km}$ wide, $60-90$ $\mathrm{m}$ deep. The Inlet cuts into the land in a south-eastern direction over a distance of about $8 \mathrm{~km}$. Its 


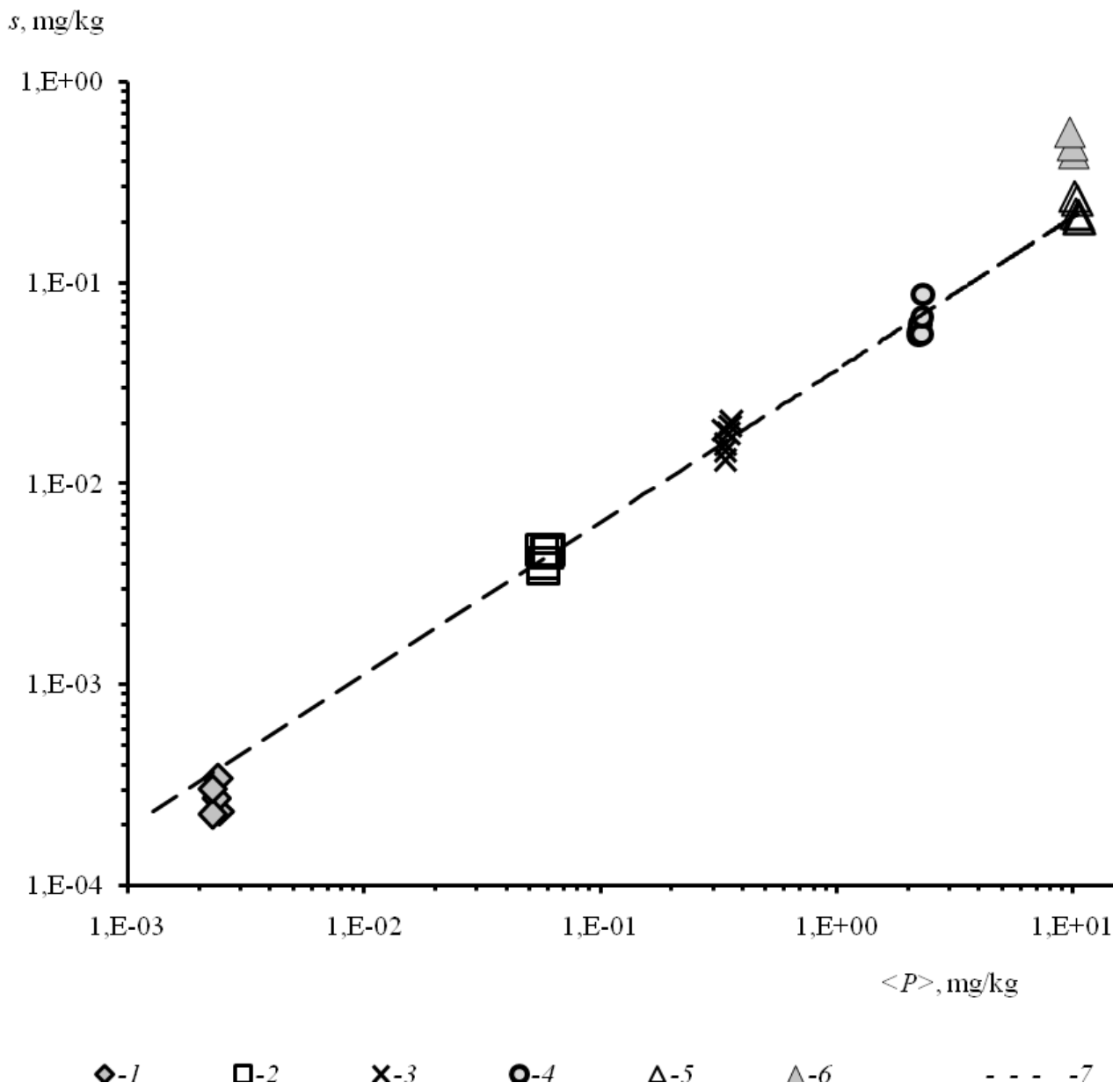

Figure 1. The relationship between the time average concentrations $\langle P\rangle$ and the sample standards $s$ for the hydrochemical parameters measured in the Ezcurra Inlet at depths of 1, 5, 10, 15, 20, 30, 40, 50, 60, and 70 m (bottom) for 0.5-2.3 months. 1-7 - nitrite, phosphate, nitrate, silicate, dissolved oxygen, dissolved oxygen only at depths of 50,60, and $70 \mathrm{~m}$, and power law regression, respectively (see initial data in [8]). Phosphates, nitrates, and silicates varying statistically insignificantly are not shown.

geographical situation and topography, in particular the steep inclination of the floor towards Admiralty Bay and further to Bransfield Strait, ensure free exchange of water with the open ocean. There is the periodical total exchange of water mass in the Inlet. There are tidal currents, superimposed surface drift currents and depth compensation currents in the Inlet. They are characterized by strong fluctuations and achieve velocities ranging from $2 \mathrm{~cm} / \mathrm{s}$ to over $40 \mathrm{~cm} / \mathrm{s}$ depending on the place, tide phase, power and direction of wind. Flow directions in upper and lower layers of water are different, sometimes opposite, varying in time. Strong vertical turbulent exchange is stimulated by flows and waves. The most probable wind velocity is $0-10 \mathrm{~m} / \mathrm{s}$, but in gusts occasionally reaches over $40 \mathrm{~m} / \mathrm{s}$. There are slight differences in water density from surface to bottom, it mean that vertical mixing takes place with particular ease [7-8]. 
The strong vertical mixing of water leads to the fact that both the time-average values of the chemical parameters $\langle P\rangle$ and the time variability $s$ at all depths are very similar in value. For this reason regression (2) is characterized by unusually small standard $s_{y x}$ and very high correlation coefficient. Judging by the content of chlorophyll $a(0.2-6.6 \mu \mathrm{g} / \mathrm{l})$ and the level of primary production $(0.15-1.50$ $\mathrm{gC} / \mathrm{m}^{2} / \mathrm{d}$ ) in the photosynthetic zone 1-50 m, photosynthesis was quite active in the Ezcurra Inlet.

However, the observed temporal nutrients variations are very slight throughout the entire vertical profile. The strong vertical turbulent mixing and the free water exchange with the open ocean are a reason for small time variations. Concurrently, silicates, nitrates and phosphates exhibit high concentrations which are similar to concentrations in Antarctic waters south of the convergence zone [7, 8, 10]. Since $\xi=s /\langle P\rangle^{\gamma}$, the parameter $\xi$ is unusually small.

The deviations of dissolved oxygen at 50,60,70 m from regression line (2) caused by a low content and by a high time variability of oxygen in comparison with the upper layer of water. The low content of oxygen $\langle P\rangle$ arises from intrusion of waters from the deeper strata of the open ocean. This water, with a salinity of over $34^{\circ} \%$ and with low oxygen content, comes from the open ocean, penetrating Admiralty Bay and further its arms easily. The observed large time fluctuations in oxygen $\xi$, with simultaneous change in salinity, are due to the tides. Near the bottom, the tidal inflows of ocean waters to the Ezcurra Inlet are most distinctly [7-8].

\section{Hydrochemical system in the Sargasso Sea}

We now consider the chemical system in the Sargasso Sea. The Fig. 2 represents the power regression obtained this paper after a preliminary analysis of daily records of nitrites, phosphates and nitrates. Only chemical parameters with diurnal variations significant at the $95 \%$ level were used for final regression. The initial data were obtained by the authors [9] on April 27-28, 1960 at six times during 24-period at depths $0,15,25,50,75,100,125,150,200$, and $300 \mathrm{~m}$ at a location 15 miles southeast of Bermuda. The observations were made, when the salinity and the temperature varied from 36.5 to $36.7^{\circ} / 00$ and from 18.0 to $21.5^{0} \mathrm{C}$ corresponding. The level of primary production $\left(0.1-0.2 \mathrm{gC} / \mathrm{m}^{2} / \mathrm{d}\right)$, the concentration of chlorophyll $a(0.04-0.81 \mu \mathrm{g} / \mathrm{l})$, and of nutrients were all as to approach the limit of sensitivity of the various methods. The final equation of a regression $s$ against $\langle P\rangle$ is:

$$
\log s=(0.89 \pm 0.08) \log \langle P\rangle+\log (0.24 \pm 0.12), \quad n=19, \quad r=0.94
$$

In the Sargasso Sea, structural features of complex hydrochemical system were as follows (Fig. 2):

1. The parameter estimate $\gamma=0.89<1$;

2. Strong correlation coefficient $r$;

3. Large scattering of points around the regression line, $s_{y x}=0.2 \mathrm{mg} / \mathrm{kg}$;

4. All points corresponding to phosphates lie below the regression line. Besides, the daily variability of phosphates in the euphotic zone at $15,25,50,75,100 \mathrm{~m}$, and at $125 \mathrm{~m}$ is not statistically significant at the $95 \%$ level;

5. Large normalization coefficient $\xi=0.24$;

6. Clearly eye-catching nitrite maximum $(0.7 \mu \mathrm{g} / \mathrm{kg})$ and variability maximum of nitrite (0.6$0.7 \mu \mathrm{g} / \mathrm{kg}$ ) at $100-125 \mathrm{~m}$. 
These features are a consequence of hydrological and biogeochemical waters regime. The waters were calm. There were no appreciable effects of hydrodynamic features. Summer conditions had just set in with the surface waters thermally stratified, nutrient impoverished, and sparsely populated with microorganisms. Those conditions were essentially those of the open Sargasso Sea [9].

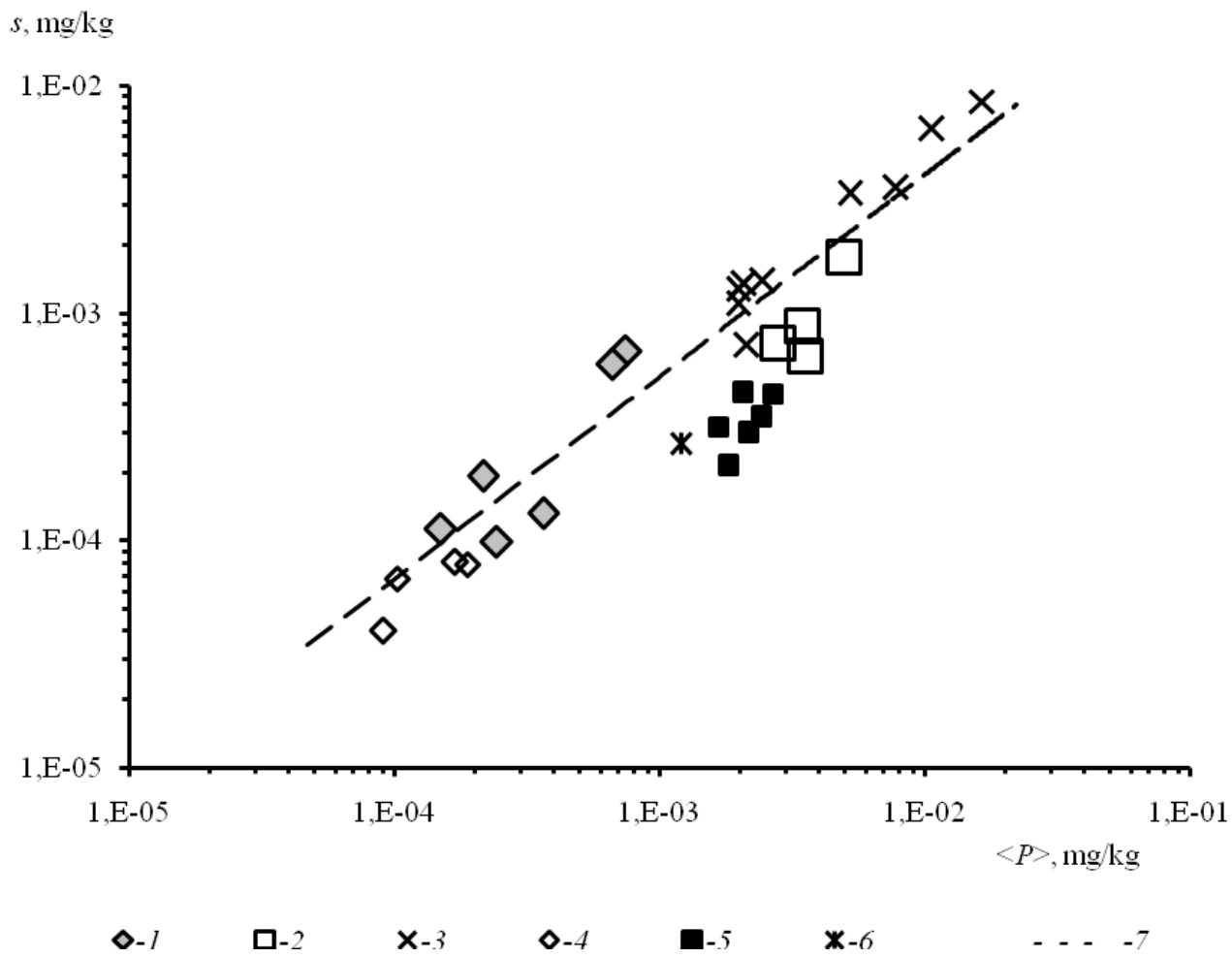

Figure 2. The relationship between the time average concentrations $\langle P\rangle$ and the sample standards $s$ for the complex of hydrochemical parameters at the daily station in the Sargasso Sea at depths of 0, 15, 25, 50, 75, 100, $125,150,200,300 \mathrm{~m} .1-3$ - nitrite, phosphate, nitrate with significant daily fluctuations, respectively; 4-6 nitrite $(15,25,50,300 \mathrm{~m})$, phosphate $(15,25,50,75,100,125 \mathrm{~m})$, nitrate $(50 \mathrm{~m})$ with insignificant at $95 \%$ level daily fluctuations, respectively; 7 - power regression (see initial data in [9]).

As intense as in the Ezcurra Inlet, vertical turbulent water mixing did not occur. Therefore, at different depths, each chemical parameter showed various pairs of $\langle P\rangle$ and $\xi$ (Fig. 2). In the surface layer, the content and variability of nutrients depended mainly on photosynthesis. Nutrient concentrations, integrated from 0 to $150 \mathrm{~m}$, increased at night and decreased during the day. Chlorophyll concentrations, integrated from the surface to $100 \mathrm{~m}$, increased during the daylight hours and decreased at night [9]. Below $150 \mathrm{~m}$, the content and variability of nutrients increased. They were apparently determined first of all by the oxidation of organic matter and advective current. All of these processes were the cause of nutrients deviations from the regression line (3). Despite the high correlation coefficient $r=0.94$, standard error of the regression reached the large value of $s_{y x}=0.20 \mathrm{mg} / \mathrm{kg}$.

The low content and low diurnal variability of phosphates and the deviations of the points from the regression line (3) are due to the consumption during the spring flowering diatoms. In the euphotic zone, at 15, 25, 50, 75, $100 \mathrm{~m}$, and at $125 \mathrm{~m}$ phosphate concentrations were the lowest and their time 
variability was insignificant. The maximum average daily phosphate content was observed outside the photic zone at $300 \mathrm{~m}(5.0 \pm 1.7 \mu \mathrm{g} / \mathrm{kg})$.

Both the concentrations $\langle P\rangle$ and the variability $s$ of nutrients were very low at the daily station. But water mixing was not intense and the time variations due to biochemical processes did not reduced. The ratio $s /\langle P\rangle^{\gamma}$ was such that the normalization coefficient was high.

Nitrite maximum and variability maximum of nitrite at 100-125 m were at the same depth as the chlorophyll maximum. A nitrite maximum is attributed to nitrate reduction by phytoplankton at low light intensities [11].

\section{Conclusion}

By the example of two sets of non-conservative chemical parameters in different regions of the World Ocean, in the Ezcurra Inlet (the Antarctic Ocean) and in the Sargasso Sea (the Atlantic Ocean), general properties and individual features of the complex hydrochemical systems structure were considered.

The Ezcurra Inlet was characterized by strong winds, intense turbulent mixing of water throughout the depth and free water exchange with the open ocean, the low water temperatures (up to $-0.4^{0} \mathrm{C}$ ), the relatively high content of chlorophyll $a$ and level of primary production, by the high concentrations, and, at the same time, the very small temporal variability of nutrients. The relative time variability of nutrients was only $3-8 \%$.

Marine environment was markedly different in the Sargasso Sea. The waters were calm. There was not vertical turbulent water mixing as intense as in the Ezcurra Inlet. The temperature varied from 18 to $22^{0} \mathrm{C}$. The surface waters were thermally stratified. The nutrients content, the chlorophyll $a$ content, and the level of primary production were very low. However, the diurnal course chlorophyll $a$ and of nutrients typical of photosynthesis was observed in the surface layer of water. The relative time variations of nutrients were $17-92 \%$.

Both hydrochemical systems, despite significant differences in the observation conditions and some difference in composition, display the collective behavior, which manifested itself in the powerlaw relationship between the time-average concentrations of non-conservative parameters and their time variability (the standard deviations). Both ratios have high correlation coefficients and similar exponents, $0.7<\gamma<0.9$. The same result was obtained earlier for the Amur Bay. The collective behavior described by the power low is the general property of the complex hydrochemical systems.

The individual features of power-low complex systems can be described by regression characteristics: normalization coefficient, standard error, and sharp deviations of the individual parts of system from the regression line and from the most probable values of characteristics of the pats, if any. The regression characteristics of complex chemical systems in the Ezcurra Inlet and in the Sargasso Sea differed significantly. It was shown that individual features of hydrochemical systems are depended on the meteorological, hydrological, hydrochemical, and hydrobiological conditions of observation. The presented regression method of analyzing the collective behavior of complex hydrochemical systems opens up new opportunities for identification their common and special properties, the water zoning, studying of the stability and the development trends, including as a hydrochemical component of ecosystems. Displaying of changes in concentrations of a large number of hydrochemical parameters in the parameters space of collective variability allows visualizing large amounts of information.

Regression analysis of the collective behavior of complex hydrochemical systems is one of the examples of the use of modern information technologies based on the methods of system analysis. 


\section{References}

[1] O.V. Shevtsova, Dokl. Earth Sci., 460, 73 (2015)

[2] B.M. Shevtsov, O.V. Shevtsova, E3S Web of Conf., 20, 02014 (2017)

[3] M.E.J. Newman, Am. J. Phys., 79, 800 (2011)

[4] O.V. Shevtsova, Water Res., 40, 166 (2013)

[5] M. Schroeder, Fractals, Chaos, Power Laws (W.H. Freeman and Company, New York, 1991)

[6] V.V. Uchaikin, Method of Fractional Derivatives (Artishok, Ul'yanovsk, 2008) (in Russian)

[7] J. Dera, Oceanologia, 12, 5 (1980)

[8] R. Bojanowski, Oceanologia, 15, 21 (1983)

[9] J.H. Ryther, D.W. Menzel, R.F. Vaccaro, Limnology and Oceanography, 6, 149 (1961)

[10] R. Hapter, B.Wozniak, Oceanologia, 15, 175 (1983) 